\title{
Dissociable contributions of the amygdala to the immediate and delayed effects of emotional arousal on memory
}

\author{
Dirk Schümann and Tobias Sommer \\ Institute for Systems Neuroscience, Medical Center Hamburg-Eppendorf, Hamburg, Germany
}

\begin{abstract}
Emotional arousal enhances memory encoding and consolidation leading to better immediate and delayed memory. Although the central noradrenergic system and the amygdala play critical roles in both effects of emotional arousal, we have recently shown that these effects are at least partly independent of each other, suggesting distinct underlying neural mechanisms. Here we aim to dissociate the neural substrates of both effects in 70 female participants using an emotional memory paradigm to investigate how neural activity, as measured by fMRI, and a polymorphism in the $\alpha_{2 B}$-noradrenoceptor vary for these effects. To also test whether the immediate and delayed effects of emotional arousal on memory are stable traits, we invited back participants who were a part of a large-scale behavioral memory study $\sim 3.5$ yr ago. We replicated the low correlation of the immediate and delayed emotional enhancement of memory across participants $(r=0.16)$ and observed, moreover, that only the delayed effect was, to some degree, stable over time $(r=0.23)$. Bilateral amygdala activity, as well as its coupling with the visual cortex and the fusiform gyrus, was related to the preferential encoding of emotional stimuli, which is consistent with affect-biased attention. Moreover, the adrenoceptor genotype modulated the bilateral amygdala activity associated with this effect. The left amygdala and its coupling with the hippocampus was specifically associated with the more efficient consolidation of emotional stimuli, which is consistent with amygdalar modulation of hippocampal consolidation.
\end{abstract}

Improved memory for emotionally arousing compared to neutral information is driven by enhanced processing and encoding as well as by improved subsequent consolidation (Hamann 2001; Ritchey et al. 2008). Preferential encoding of emotional stimuli results immediately in enhanced memory and is based on their tendency to attract greater attention, resulting in deeper processing (Kensinger and Corkin 2004; Kang et al. 2014). This affect-biased attention, attributed to amygdala and central noradrenergic system activity, has been suggested to increase signal-to-noise ratio in sensory areas and synaptic plasticity in the hippocampus (Markovic et al. 2014; Mather et al. 2015; Hagena et al. 2016), leading to better encoding. The amygdala along with the central noradrenergic system also modulate the delayed effect of emotional arousal on consolidation (McIntyre et al. 2012; Manns and Bass 2016; Inman et al. 2017). This delayed effect of arousal on consolidation can be explained by the tagging of active synapses during the processing of emotional stimuli that results in the subsequent transformation of early in persistent late long-term potentiation and hence improvement in consolidation (Bergado et al. 2011; McIntyre et al. 2012).

To investigate the relationship between the emotional enhancement in immediate (encoding) and delayed (consolidation) memory processes and the genetic predisposition that could influence affect-biased attention, we previously conducted a large-scale behavior-genetic study $(n=690)$. We observed that the magnitude of the effects of arousal on encoding and consolidation, i.e., immediate emotional enhancement of memory (iEEM) and delayed emotional enhancement of memories (dEEM), correlated only weakly with each other across participants $(r=0.14)$ (Schümann et al. 2017). In some participants, emotional arousal only en-

\section{Corresponding author: tsommer@uke.de}

Article is online at http://www.learnmem.org/cgi/doi/10.1101/lm.047282.117. hanced encoding (better memory for emotional items 10 min after encoding) but did not result in more efficient consolidation (memory for emotional and neutral items were similar $20 \mathrm{~h}$ after encoding), whereas others showed the opposite pattern, i.e., only an effect on consolidation (better memory for emotionally arousing than neutral items after $20 \mathrm{~h}$ but not immediately). The relative independence of these two effects from each other is consistent with these effects having partly distinct underlying processes and neurobiological substrates although the central noradrenergic system and the amygdala play critical roles in both effects. The same mechanisms in these brain structures initiate both effects, but then the processes diverge, resulting in iEEM and dEEM. One possibility is the central noradrenergic system and amygdala interact with different brain areas to modulate signal-to-noise ratio in sensory areas or synaptic plasticity and synaptic tagging in the hippocampus. Alternatively, the processes resulting in iEEM and dEEM might diverge as early as in the amygdala, whether in different parts of the amygdala or on the molecular level within the same area.

Three previous fMRI studies had aimed to disentangle the neural substrates of iEEM and dEEM (Mackiewicz et al. 2006; Ritchey et al. 2008; Mickley Steinmetz et al. 2012), but results are not fully consistent and open questions remain. Using an immediate and a delayed recognition test of negative compared to neutral pictures (i.e., the main effect of emotion on memory), Mackiewicz et al. (2006) found that bilateral dorsal amygdala was related to

(C) 2018 Schümann and Sommer This article is distributed exclusively by Cold Spring Harbor Laboratory Press for the first 12 months after the full-issue publication date (see http://learnmem.cshlp.org/site/misc/terms.xhtml). After 12 months, it is available under a Creative Commons License (AttributionNonCommercial 4.0 International), as described at http://creativecommons. org/licenses/by-nc/4.0/. 
iEEM in both men and women, whereas dEEM elicited activity in the left ventral amygdala in women and the right in men. The other two studies used subsequent memory effect (SME) analyses (Dolcos et al. 2012) to identify areas where trial-wise neural activity during encoding was correlated with iEEM or dEEM. The greater persistence of emotional memories, i.e., that dEEM is often behaviorally stronger than that of iEEM, was not related to greater activity in the amygdala but increased coupling of the amygdala and the parahippocampal cortex (Ritchey et al. 2008). This study did not aim to identify which brain areas were more involved in iEEM. Testing the hypothesis that the subsequent memory effect for emotional items is more delay-invariant than for neutral items, Mickley Steinmetz et al. (2012) indeed found that more cortical areas correlated with immediate than delayed subsequent memory for only neutral, not negative, items. However, no activity related to either iEEM or dEEM in the amygdala was observed.

In the study we present here, we aimed to complement the findings of these previous studies by directly comparing the neural correlates of iEEM and dEEM, i.e., the interaction of immediate and delayed subsequent memory effects (SMEs), respectively. In particular, participants studied 80 neutral and 80 negatively arousing scenes in the MRI scanner, completed then a recognition test for half of the pictures outside of the scanner and a second recognition test for the remaining pictures followed $\sim 24 \mathrm{~h}$ later. As the difference between the iEEM and AEEM can be considered in terms of affect-biased attention (i.e., iEEM) versus emotional synaptic tagging (i.e., dEEM), and we know that affect-biased attention and emotional synaptic tagging rely on amygdala modulation of different brain areas, we focused on the functional coupling of the amygdala with other brain areas related to iEEM and dEEM. Such direct comparisons of iEEM and dEEM is challenging because many emotionally arousing items involve both enhanced attention and synaptic tagging, though our previous study suggests that both processes are relatively independent of each other (Schümann et al. 2017). These items cannot be identified and then excluded from the analyses because each item can be tested only once, immediately or delayed (otherwise the second test would be confounded by the first). These confounded items can only be treated as noise when one aims to separate the neural correlates of iEEM and dEEM during initial processing. To overcome this limitation inherent to the nature of EEM, we recruited a relatively large sample of 70 participants in an attempt to increase statistical power needed to detect activity uniquely associated with iEEM or with dEEM. Only female participants were recruited since lateralized amygdala activ- ity related to dEEM has been reported to depend on sex (Cahill et al. 2004; Mackiewicz et al. 2006).

In our previous large-scale behavioral study, we had tested whether iEEM and dEEM's partial independence of each other could be explained by the differential involvement of the adrenoceptor subtypes, which vary in affinity, action, and expression pattern (Hein 2006; Luhrs et al. 2016). Participants were genotyped for a polymorphism in the gene that codes for the $\alpha_{2 \mathrm{~B}}$-noradrenergic receptor, a polymorphism that has been associated with greater perceived vividness of emotional stimuli, affect-biased attention, and iEEM in free recall (de Quervain et al. 2007; Rasch et al. 2009; Todd et al. 2013, 2015). However, we did not find any relationship between the polymorphism and iEEM or dEEM behaviorally (Schümann et al. 2017). Given that differences in neural activity are not necessarily behaviorally observable, we invited back a subset of the genotyped participants from our previous large-scale behavioral study in order to investigate whether $\alpha_{2 \mathrm{~B}}$-noradrenoceptor polymorphism is associated with differences in neural activity between iEEM and dEEM. This also enabled us to investigate to what degree iEEM and dEEM are stable personality traits, which would in turn suggest genetic influences.

\section{Results}

\section{Behavioral results}

Hits were defined as a response of three or lower on old items on the six-step confidence rating during the recognition test (six high confidence new, one high confidence old), and misses as responses of four or higher. Corrected hit rates (hit rate-false alarm rate) were higher for negative than for neutral stimuli at both retention intervals (Fig. 1, left panel). A GLM with the within-subject factors of retention interval and valence revealed main effects of retention interval, $F_{(1,68)}=306.98, P<0.0001, \eta^{2}=0.24$, and valence, $F_{(1,68)}=$ 83.08, $P<0.0001, \eta^{2}=0.07$, and an interaction between these two factors, $F_{(1,68)}=29.32, P<0.0001, \eta^{2}=0.01$, indicating that the effect of emotional arousal was larger during consolidation (with delay). There was no correlation between delayed (dEEM) and immediate (iEEM) effects of emotional arousal: $r=0.16(P=0.20$; Fig. 1 , middle panel), which replicates our earlier finding. To give a full picture of the behavioral results, we also computed d-prime, response bias (c), and, as a pure measure of accuracy (Dougal and Rotello 2007), the area under the curve (AUC) of the ROC-curves based on the confidence ratings (Table 1). According to the dual
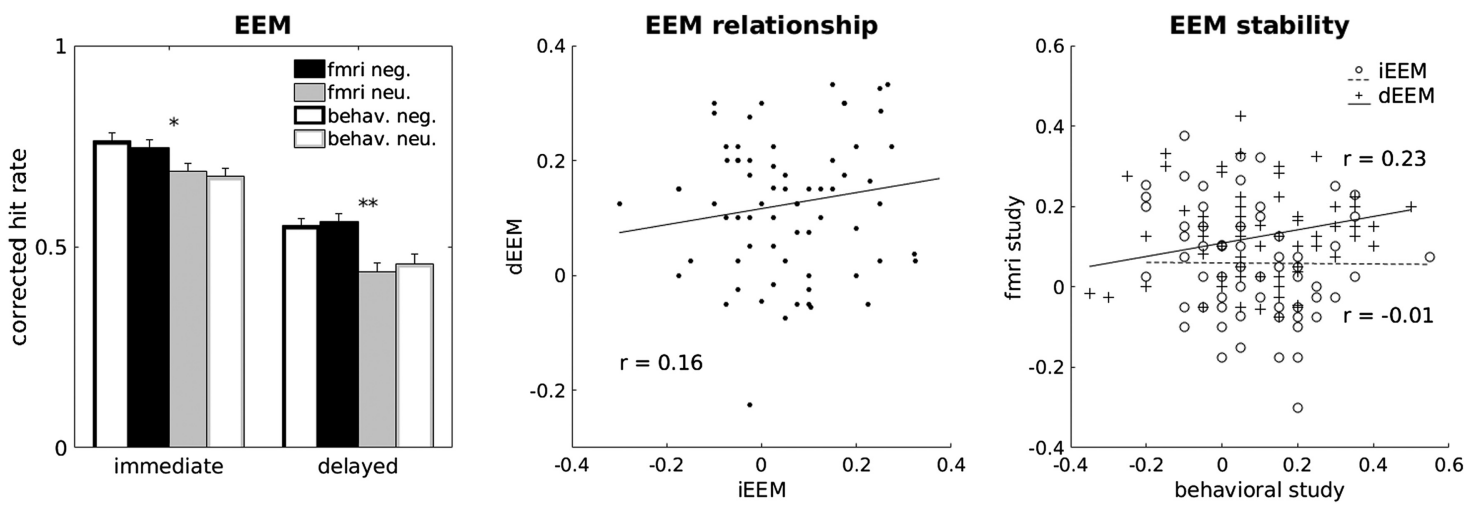

Figure 1. Behavioral results. (Left panel) Corrected hit rates in the immediate and delayed memory test for negative (black) and neutral (gray) pictures from the current study and from the previous large-scale behavioral study of the same participants (solid bars-current fMRI study, contour bars-largescale behavioral study). (Middle panel) Correlations between the immediate and delayed emotional enhancement of memory (iEEM and dEEM, respectively) across participants in the current study. Exclusion of the outlier changes correlation to $r=0.14$. (Right panel) Correlations of iEEMs and of dEEMs between the current study and the previous large-scale behavioral study of the same participants. 
Table 1. Behavioral results in the immediate and delayed recognition tests

\begin{tabular}{lccccccc}
\hline & Hit rate & False alarm rate & c & $\mathbf{d}^{\prime}$ & Familiarity & Recollection & AUC \\
\hline Immediate test & & & & & & & \\
$\quad$ Negative & $0.85(0.12)$ & $0.11(0.09)$ & $0.17(0.43)$ & $2.78(1.26)$ & $1.63(0.86)$ & $0.53(0.29)$ & $0.92(0.07)$ \\
$\quad$ Neutral & $0.81(0.13)$ & $0.13(0.09)$ & $0.10(0.50)$ & $2.42(1.06)$ & $1.43(0.66)$ & $0.46(0.26)$ & $0.89(0.08)$ \\
Delayed test & & & & & & \\
$\quad$ Negative & $0.68(0.15)$ & $0.12(0.09)$ & $0.43(0.45)$ & $1.91(0.80)$ & $1.32(0.53)$ & $0.28(0.21)$ & $0.84(0.08)$ \\
$\quad$ Neutral & $0.61(0.19)$ & $0.17(0.11)$ & $0.37(0.43)$ & $1.38(0.66)$ & $0.93(0.51)$ & $0.20(0.20)$ & $0.76(0.11)$ \\
\hline
\end{tabular}

Familiarity and recollection were computed based on the dual process model of recognition memory (Koen et al. 2016, c response criterion, AUC area under the curve, mean, and standard deviation).

process model of recognition memory, two independent processes contribute to recognition, where "recollection" refers to the retrieval of an item together with contextual information about the encoding episode in contrast to the acontextual sense of "familiarity" (Yonelinas 2002). To assess the contribution of recollection and familiarity to the EEMs, the dual process model of recognition memory was fit to the individual data using the ROC toolbox (Koen et al. 2016).

The mean magnitude of both effects for participants was similar across the current study and when they participated in the previous large-scale behavioral study (Fig. 1, left panel; GLM: main effect of study: $F_{(1,68)}=0.07, P=0.79$; no interactions between the factors study, valence and delay, all $P^{\prime} \mathrm{s}>0.10$ ). Most important, all post-hoc tests contrasting the same condition across studies, e.g., immediate corrected hit rate for neutral pictures, were not significant (all $P$ 's $>0.40$ ). Surprisingly, individual iEEM did not correlate across both studies $(r=-0.01, P=0.95)$ and the correlation was weak for $\operatorname{dEEM}(r=0.23, P=0.06)$. However, general mnestic ability, i.e., stability of memory performance across studies within each of the four conditions, was substantially higher than the EEMs (corrected hit rates of immediate neutral $r=0.59$, immediate negative $r=0.40$, delayed neutral $r=0.39$, delayed negative $r=0.42$, see Table 2 for the correlations on additional memory measures).

Genotype had no effect on behavioral measures of iEEM of dEEM. A mixed GLM with delay and valence as within subject factors and genotype as between subject factor showed no effects for genotype and no genotype interaction for the adrenergic polymorphism (all $P^{\prime}$ s $>0.31$ ).

\section{fMRI results}

We adopted both an ROI-(left and right amygdala and hippocampus) as well as a peak-based approach to characterize brain activity associated with iEEM and dEEM. The ROI-based analyses revealed a main effect of brain structure, with greater EEMs in the amygdala than hippocampus $\left(F_{(1,67)}=24.70, P<0.00001\right.$; Fig. 2$)$. There was also an interaction of brain structure with delay: iEEM involved the hippocampus less than the amygdala, whereas the dEEM involved both structures $\left(F_{(1,67)}=4.71, P=0.03\right.$; post-hoc Tukey HSD: amygdala iEEM greater than hippocampus iEEM $P<0.01$ ). Additionally, the three-way interaction reached significance $\left(F_{(1,67)}=4.95, P=0.03\right)$. To explore the differences leading to this three-way interaction, post-hoc GLMs with iEEM and AEEM as dependent variables and the within subject factors hemisphere and brain structure were computed. This GLM revealed a main effect of brain structure for iEEM, confirming that stronger iEEM is more associated with the amygdala than the hippocampus $\left(F_{(1,67)}=24.93, P<0.00001\right)$. The corresponding GLMs for dEEM revealed a trend toward larger dEEMs in the amygdala than the hippocampus $\left(F_{(1,67)}=3.13, P=0.08\right)$ and a significant interaction suggesting a smaller dEEM in the right compared to left amygdala $\left(F_{(1,67)}=4.83, \quad P=0.03\right.$; post-hoc Tukey HSD: right amygdala dEEM greater than left amygdala dEEM $P=0.02$ ).

The peak voxel-based approach identified a wide network of brain regions, including the amygdala, was more active during processing negative than neutral pictures (Table 3). Several of the previously reported brain regions were associated with iEEM and dEEM, with dEEM eliciting activity in more areas overall (Table 3). Notably, activity in the bilateral amygdala was associated with iEEM whereas dEEM only correlated with activity in the left amygdala/anterior hippocampus (Fig. 3). This was confirmed by the exclusive masking procedure which revealed bilateral anterior amygdala for iEEM ( $x y z=\left[\begin{array}{lll}18 & 0 & -16\end{array}\right], Z=4.39 ; x y z=\left[\begin{array}{lll}-20 & -2\end{array}\right.$ $-20], Z=3.56$, Fig. 3) and left posterior amygdala/anterior hippocampus for dEEM (xyz $=[-26-12-14], Z=4.04$, Fig. 3$)$. The interaction analysis revealed no surviving clusters of activity for iEEM, with the lowest $P$-value in the right amygdala $(\mathrm{Z}=2.81, P=0.17)$, and for AEEM, clusters in the left inferior and middle frontal gyrus survived whole brain FWE correction (xyz $=\left[\begin{array}{lll}-48 & 26 & 18\end{array}\right]$, $\left.\mathrm{Z}=4.82, P=0.24 ; \mathrm{xyz}=\left[\begin{array}{lll}-30 & 6 & 40\end{array}\right], \mathrm{Z}=4.77, P=0.030\right)$. The conjunction analysis revealed a significant cluster in the left amygdala $(\mathrm{xyz}=[-18-4-14], \mathrm{Z}=3.77, P=0.01)$.

Table 2. Correlations of memory performance measures of the same 69 participants between the large-scale behavioral study $3.5 \mathrm{yr}$ ago and the current fMRI study

Correlation large-scale behavioral study $\times$ current fMRI study

\begin{tabular}{|c|c|c|c|c|c|c|c|c|}
\hline & Corrected hit rate & Hits & False alarms & $\mathbf{d}^{\prime}$ & c & fam & $\operatorname{Rec}$ & AUC \\
\hline \multicolumn{9}{|c|}{ Immediate test } \\
\hline Negative & $0.40^{* *}$ & $0.37^{* *}$ & $0.33^{* *}$ & $0.33^{* *}$ & 0.20 & 0.03 & 0.19 & $0.37^{* *}$ \\
\hline Neutral & $0.59^{\star *}$ & $0.55^{* *}$ & $0.58^{\star *}$ & $0.41^{\text {** }}$ & $0.41^{* *}$ & 0.14 & $0.35^{\star *}$ & $0.58^{\star *}$ \\
\hline EEM & -0.01 & 0.13 & 0.15 & -0.13 & 0.16 & 0.07 & 0.18 & 0.10 \\
\hline \multicolumn{9}{|l|}{ Delayed test } \\
\hline Negative & $0.42^{* *}$ & $0.36^{* *}$ & $0.50^{* *}$ & $0.42^{* *}$ & $0.34^{* *}$ & 0.18 & -0.05 & $0.41^{\text {** }}$ \\
\hline Neutral & $0.39^{* *}$ & $0.35^{* *}$ & $0.41^{\star *}$ & $0.43^{* *}$ & $0.39^{* *}$ & $0.30^{*}$ & $0.61^{* *}$ & $0.48^{* *}$ \\
\hline EEM & $0.23^{\mathrm{t}}$ & $0.35^{\star *}$ & 0.06 & 0.08 & 0.21 & -0.08 & 0.18 & 0.22 \\
\hline
\end{tabular}

Rec, recollection; fam, familiarity according to the dual process model of recognition memory (Koen et al. 2016); EEM, Emotional Enhancement of Memory computed as differences between negative and neutral performance. ${ }^{*} P<0.05 ;{ }^{* *} P<0.01 ;{ }^{t} P<0.10$. 


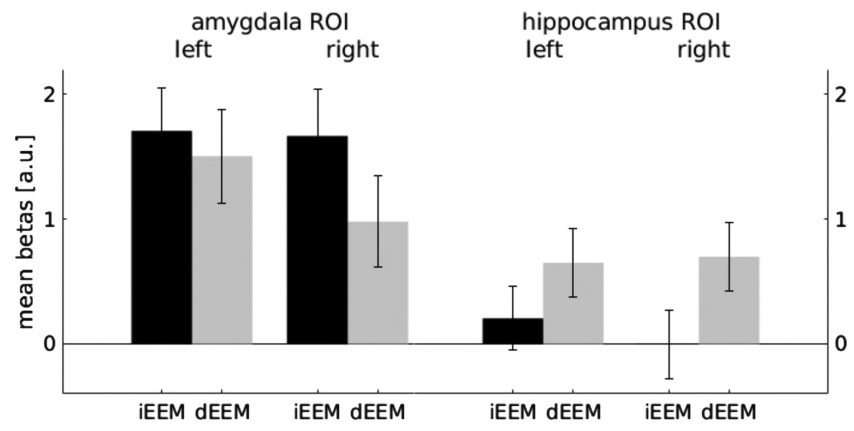

Figure 2. Mean amygdala and hippocampal activity associated with iEEM and dEEM: Results of the ROI-based analyses. Mean activity across all voxels in the left and right amygdala and side-matched hippocampus ROIs. Immediate and delayed EEM are shown.

The psychophysiological interaction (PPI) analyses using a region in the left amygdala as a seed, as identified by the main effect of emotion, revealed stronger coupling during successful encoding of negative than neutral pictures with large clusters in visual areas for iEEM (Table 3; Fig. 4). During processing that was associated with more efficient consolidation for negative than neutral pictures, the amygdala was coupled more strongly with these visual areas as well as with the bilateral hippocampus and inferior frontal gyrus. The exclusive masking procedure revealed that clusters in visual areas, in particular in the fusiform $(x y z=[28-58-6], Z=4.59$, $x y z=[-26-64-4], Z=5.17)$, lingual $(x y z=[26-64-6], Z=4.10$, $\mathrm{xyz}=[-22-880], Z=4.85)$, and middle occipital gyrus $(\mathrm{xyz}=[22$ $-880], Z=4.78$ ) were predominantly related to iEEM, whereas the hippocampus and inferior frontal gyrus to dEEM.

The $\alpha_{2 \mathrm{~B}}$-noradrenergic receptor deletion genotype (32 wildtype, 37 deletion carriers) was associated with the greater activity in the medial PFC, bilateral hippocampus and visual cortex (Table 2) for the main effect of emotion, where deletion carriers showed greater activity in these areas for negative pictures. However, the largest $\mathrm{p}$-value in the amygdala was only $P=0.29(\mathrm{Z}=2.54)$ at $\mathrm{xyz}=[-30-6-28]$. There were no brain areas associated with greater activity for participants with the wild-type of this adrenoceptor compared to the deletion carriers. However, wild-type carriers had greater iEEM-related activity in the bilateral amygdala, insula, and mPFC/anterior cingulate (Table 3; Fig. 5). Deletion carriers did not show any greater activity associated with iEEM. For dEEM, neither group, whether deletion carriers or wild-type, showed significantly greater activity.

\section{Discussion}

Recognition memory for negatively emotional pictures was better than for neutral pictures both immediately and after $24 \mathrm{~h}$, where this advantage increased after the consolidation delay, replicating previous findings, including from our previous large-scale behavioral study (Sharot et al. 2007; Payne et al. 2008; Sharot and Yonelinas 2008; Schümann et al. 2017). Also similar to the previous findings from our large-scale behavioral and a smaller replication study, we found only a weak correlation between individual iEEM and dEEM (Schümann et al. 2017). The consistently low correlation between iEEM and dEEM supports the hypothesis that both effects of emotional arousal, reflecting affect-biased attention and emotional synaptic tagging, respectively, rely at least partially on distinct neural substrates.

The mean performance in the four conditions (i.e., immediate negative/neutral, delayed negative/neutral) was similar to our large-scale behavioral study $\sim 3.5 \mathrm{yr}$ earlier and was stable over time across participants, suggesting the trait-like quality and even inheritability of general mnestic ability (McClearn et al. 1997; Panizzon et al. 2011). The mean iEEM and dEEM across participants of the current study was also similar to the large-scale behavioral study, but only a weak correlation for individual dEEM across studies was found. It should be reiterated that although the timing, including the active baseline task, of the emotional memory paradigms in the two studies were identical, no emotionally positive pictures were included in the current study (whereas participants encoded 40 positive, 40 neutral as well as 40 negative pictures in the previous study). Moreover, participants in the current study encoded in the MR scanner but retrieved on a computer outside of the scanner, which might have led to greater stress during encoding and to less context-dependent memory during recognition. The low correlations in within-subject iEEMs and dEEMs across the two studies might, therefore, be affected by such rather subtle differences in task and settings. We did not assess the hormonal status of the participants (menstrual cycle phase and use of oral contraceptives) because we did not observe an effect of hormonal status on memory or EEMs in 464 women $(62.5 \%$ using oral contraceptives) in the previous large-scale behavioral study (unpublished observation). Similarly, we did not observe an effect of hormone status on memory or EEMs, nor on amygdala activity, in a recent study where we pharmacologically increased estrogen levels across a wide range in 125 naturally cycling women (Bayer et al. 2018). However, another previous study using a more sensitive within-subject design contrasting natural cycling women in their early follicular and luteal phase showed subtle differences in emotional memory performance (Bayer et al. 2014). Differences in hormonal status might have therefore also reduced the correlations of the EEMs across studies. However, these differences unlikely account for all of the individual variances; the low correlations might also suggest low retest reliability and/or stability of iEEM and dEEM. In other words, the typical paradigm used to assess the effects of emotional arousal on encoding and consolidation is either not well suited to measure potentially underlying personality traits, or iEEM and dEEM are not traits, but rather, states.

Both interpretations are of relevance for behavior-genetic studies on EEM (Todd et al. 2011) and might explain why we previously failed to replicate a genetic influence of $\alpha_{2 \mathrm{~B}}$-adrenoceptor polymorphism on iEEM (Schümann et al. 2017). Importantly, previous studies reporting a influence of this polymorphism on iEEM used free recall (Rasch et al. 2009; de Quervain et al. 2007) where iEEM does not primarily depend on affect-biased attention during encoding but, to a larger degree, on processes during recall such as semantic relatedness and distinctiveness (Sommer et al. 2008; Barnacle et al. 2016; Talmi et al. 2017). The low retest reliability/ stability of the EEMs is also more generally of interest because many behavior and/or imaging genetic studies use cognitive psychological measures, e.g., difference in performance between two conditions, with unknown psychometric characteristics as phenotype.

The ROI-based analysis of amygdala and hippocampal activity revealed an interesting functional differentiation along the anterior-posterior axis: amygdala, but not hippocampus activity, was correlated with iEEM, whereas the hippocampus was involved also in dEEM. Moreover, the data suggest a lateralization of the amygdala, with activity in the left with larger dEEM. It is important to keep in mind that we cannot rule out that the overlap of both effects in the left amygdala might be caused by the unavoidable confound that many of the items contributing to dEEM also involved more affect-biased attention (iEEM) during encoding. Taken together, however, the ROI-based analyses suggest that the neural correlates of iEEM and AEEM can be differentiated along an anterior-posterior gradient from the amygdala to the hippocampus, as well as a left-right gradient across the amygdalae. 
Table 3. fMRI results

Peak coordinate

\begin{tabular}{|c|}
\hline $\begin{array}{l}\text { Main effect emotion } \\
\text { Amygdala }\end{array}$ \\
\hline $\begin{array}{l}\text { vmPFC } \\
\text { mPFC/anterior cingulate } \\
\text { Inferior frontal }\end{array}$ \\
\hline Insula \\
\hline Thalamus \\
\hline $\begin{array}{l}\text { Mid-posterior cingulate } \\
\text { Middle temporal }\end{array}$ \\
\hline Fusiform \\
\hline Supramarginal \\
\hline $\begin{array}{l}\text { iEEM } \\
\text { Amygdala }\end{array}$ \\
\hline $\begin{array}{l}\mathrm{mPFC} / \text { anterior cingulate } \\
\text { Insula }\end{array}$ \\
\hline Thalamus \\
\hline Middle temporal \\
\hline $\begin{array}{l}\text { Temporal pole } \\
\text { dEEM } \\
\text { Hippocampus/amygdala } \\
\text { mPFC/anterior cingulate }\end{array}$ \\
\hline $\begin{array}{l}\text { Superior frontal } \\
\text { Middle frontal }\end{array}$ \\
\hline Insula \\
\hline $\begin{array}{l}\text { Thalamus } \\
\text { Caudate }\end{array}$ \\
\hline Inferior parietal \\
\hline Inferior frontal \\
\hline Inferior temporal \\
\hline $\begin{array}{l}\text { iEEM PPI with amygdala as seed } \\
\text { Fusiform }\end{array}$ \\
\hline Lingual \\
\hline Middle occipital \\
\hline $\begin{array}{l}\text { dEEM PPI with amygdala as seed } \\
\text { Hippocampus }\end{array}$ \\
\hline Fusiform \\
\hline Lingual \\
\hline Middle occipital \\
\hline Inferior frontal \\
\hline
\end{tabular}

$20-6-12$
$-20-6-14$
$-248-18$
-65020
52384
-44280
$3412-20$
$-308-20$
$6-240$
$-4-142$
$-4-4828$
$50-60-2$
$-52-644$
$42-68-12$
$-40-50-18$
$62-2230$
$-62-2830$

$R$

R

L

R

$\mathrm{L}$

R

L

$\mathrm{L}$

L

$R$
$L$

$-18-2-14$
10406

R $\quad 3218-12$

$3218-12$
$4010-10$

$-3612-6$

$4-100$

$-12-46$

$58-24-10$

$-46-6014$

$-3812-22$

$-22-8-16$

10466

$-24424$

$-10406$

62450

$-302838$

$-36642$

401038

$38-12-4$

$4216-8$

$-3820-2$

$2-124$

$-1066$

$16-2-18$

$62-3836$

$-58-3238$

$5226-4$

$-3828-8$

$56-62-4$

$-46-36-20$

$28-72-10$

$-26-64-8$

$14-90-2$

$-22-86-8$

$32-862$

$-28-886$

$28-34-2$

$-20-28-8$

$38-52-20$

$-26-60-14$

$-32-78-16$

$42-6810$

$-38-8810$

521822

$-521614$
$-58-60-10$

$24-82-12$

Z (peak coordinate)

Cluster size

Cluster size exclusively masked

Inf 263

Inf 243

$\begin{array}{lr}5.8 & 316 \\ \operatorname{lnf} & 3699\end{array}$

Inf 1828

Inf 1347

$5.71 \quad 22$

$\begin{array}{rr}5.49 & 70 \\ 6.49 & 240\end{array}$

$5.61 \quad 124$

Inf 1966

Inf 1132

Inf 1086

Inf 506

Inf $\quad 525$

$7.46 \quad 414$

$6.53 \quad 464$

$\begin{array}{ll}4.50 & 128\end{array}$

$\begin{array}{ll}4.18 & 127 \\ 3.89 & 324\end{array}$

$3.89324^{*}$

$\begin{array}{ll}4.26 & 28^{\star} \\ 3.65 & 24^{\star}\end{array}$

$3.6613^{\star}$

$3.8488^{\star}$

3.4848

3.87

4.14

4.91

4.46

4.13

4.57

4.77

5.55

4.38

4.28

4.24

4.80

4.11

4.28

5.53

5.21

4.43

5.10

4.30

5.81

4.82

4.46

4.03

3.98

5.96

6.08

5.17

5.13

5.33

5.83

4.41

4.88

4.59

4.69

4.15

4.69

4.39

5.02

3.73

4.76
17
7

163

336
209

73

201

1447

150

132

35

105

120
65

55

832

404
1571

90

147

27
34

34
19

40
59

121

72

158

Continued 
Table 3. Continued

\begin{tabular}{|c|c|c|c|c|c|}
\hline & & Peak coordinate & Z (peak coordinate) & Cluster size & $\begin{array}{c}\text { Cluster size } \\
\text { exclusively masked }\end{array}$ \\
\hline \multicolumn{6}{|c|}{$\alpha_{2 \mathrm{~B}}$-adrenoceptor genotype effect (deletion $>$ wild-type) on the main effect of emotional arousal } \\
\hline $\mathrm{mPFC} /$ anterior cingulate & & 44420 & 4.80 & 1050 & \\
\hline \multirow[t]{2}{*}{ Hippocampus } & $\mathrm{R}$ & $30-32-8$ & 4.55 & 108 & \\
\hline & L & $-22-28-10$ & 3.76 & 53 & \\
\hline \multirow[t]{3}{*}{ Insula } & $\mathrm{R}$ & $40-14-2$ & 3.85 & $18^{*}$ & \\
\hline & & $402-16$ & 3.82 & $23^{*}$ & \\
\hline & L & -3622 & 3.54 & $14^{*}$ & \\
\hline Calcarine & $\mathrm{R}$ & $12-726$ & 4.55 & $76^{*}$ & \\
\hline \multicolumn{6}{|c|}{$\alpha_{2 \mathrm{~B}}$-adrenoceptor genotype effect (wild-type > deletion) on the iEEM } \\
\hline \multirow[t]{2}{*}{ Amygdala } & $\mathrm{R}$ & $26-2-20$ & 4.45 & 104 & \\
\hline & $\mathrm{L}$ & $-30-6-18$ & 3.66 & 38 & \\
\hline \multirow[t]{2}{*}{ Insula } & $\mathrm{R}$ & $362-12$ & 5.12 & 101 & \\
\hline & $\mathrm{L}$ & $-36-10-8$ & 4.69 & $98^{*}$ & \\
\hline $\mathrm{mPFC} /$ anterior cingulate & & -22022 & 4.52 & 172 & \\
\hline
\end{tabular}

The number of voxels of activity clusters that survived the exclusive masking procedure with the opposite contrast are listed to show which clusters were predominantly related to iEEM or dEEM. Peaks with a $P<0.001$ uncorrected for multiple comparisons and minimum 10 voxel cluster extant are marked with a * (Cluster inducing threshold of $P<0.001$ uncorrected).

This varying degree of involvement of the amygdalae and hippocampus for iEEM and dEEM would be consistent with their relative independence of each other, as shown by their low correlation with each other (Schümann et al. 2017).

Results from the peak-based analyses, providing higher spatial specificity, and subjected to the exclusive masking procedure, generally confirmed this pattern. In particular, a cluster in the right amygdala was correlated only with iEEM, a cluster in the left amygdala with both iEEM and dEEM, and a cluster in the left posterior amygdala/anterior hippocampus correlating only with dEEM. We did not find (with the given spatial resolution) evidence for a functional differentiation in the central, more dorsal, amygdala previously suggested to be involved in iEEM, and basolateral, more ventral, amygdala involved in dEEM (Mackiewicz et al. 2006). Instead, our data show an overlap of left amygdala activity associated with both effects, similar to other reports (Ritchey et al. 2008) and may suggest, if anything, an anterior-posterior dissociation. It should also be noted that the more rigid statistical interaction tests could not dissociate lateral contributions of the amygdala to dEEM nor confirm the exclusive contribution of the hippocampus to dEEM and not to iEEM.

The suggested functional lateralization of the amygdala would be consistent with previous reports of the sex-dependent lateralization of dEEM. Specifically, the left amygdala contributes to dEEM only in women, whereas the bilateral amygdala is associated with the iEEM in both sexes (Cahill et al. 2001, 2004; Canli et al. 2002; Kilpatrick and Cahill 2003; Mackiewicz et al. 2006). Alternatively, the right amygdala was claimed to be part of a rapid emotional stimulus detection system, which would be consistent with a role in affect-based attention, whereas the left amygdala might be specialized for more sustained responses (Phillips et al. 2001; Wright et al. 2001;
Sergerie et al. 2008; Kohno et al. 2015). In line with a functionaland/or sex-dependent lateralization of amygdala activity, rodents show an anatomical lateralization of the amygdala which is in part sex-dependent (Johnson et al. 2008, 2012; Pfau et al. 2016).

Not only did activity in the amygdala differ between iEEM and AEEM, but its functional coupling with other cortical areas were also modulated depending on the EEM. In particular, the amygdala was functionally more strongly coupled with visual areas and the fusiform gyrus for iEEM and AEEM, and additionally with the hippocampus for dEEM. Both activity patterns overlapped,
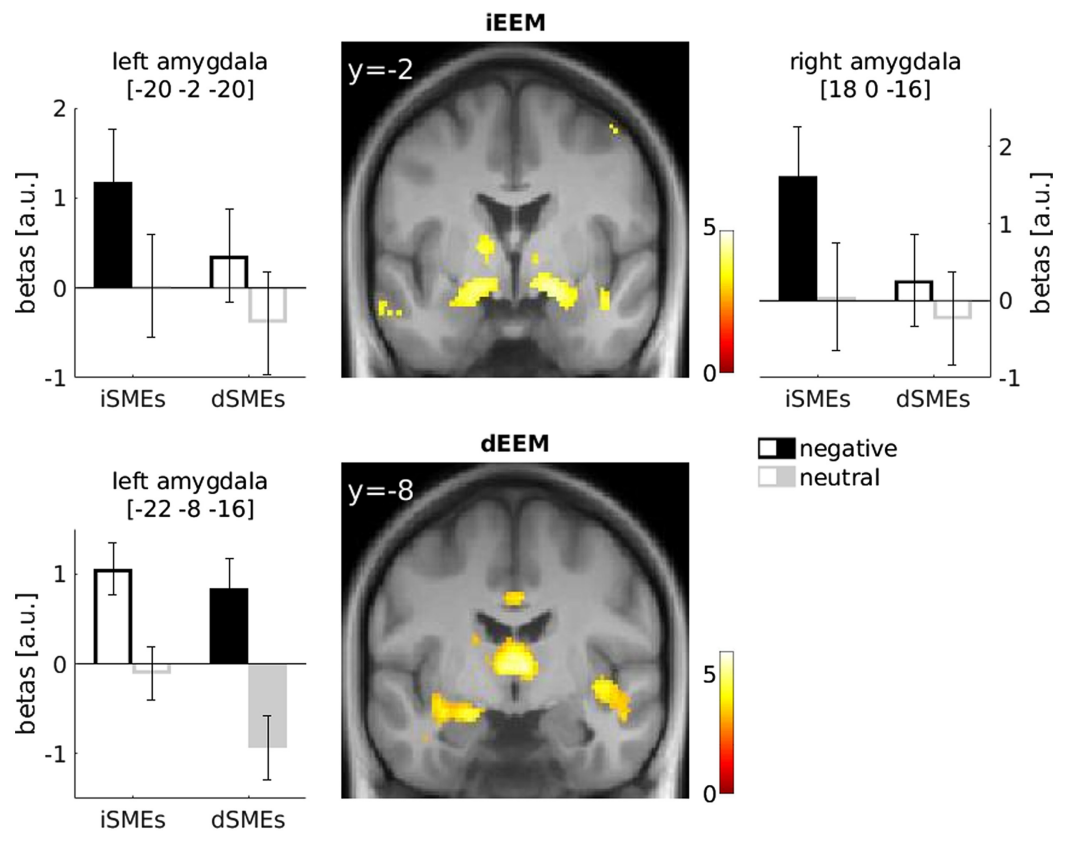

negative neutral

Figure 3. Amygdala activity associated with iEEM and dEEM: Results of the peak voxel-based analyses. (Upper panel) Activity in the bilateral amygdala correlated with iEEM. (Lower panel) Activity in the left amygdala/anterior hippocampus correlated with dEEM. Negative and neutral SMEs, i.e., activity during subsequent hits minus misses, in the immediate (iSMEs) and delayed (dSMEs) tests are shown. Importantly, parameter estimates are shown only for the peaks that survived the exclusive masking with the opposing EEM contrast and therefore represent the largest observed differences between activities associated with iEEM and dEEM in the given brain areas. The solid bars represent the effect (iEEM or $\mathrm{dEEM}$ ) that was significant in the area plotted, the contour bars the effects used as exclusive mask. 

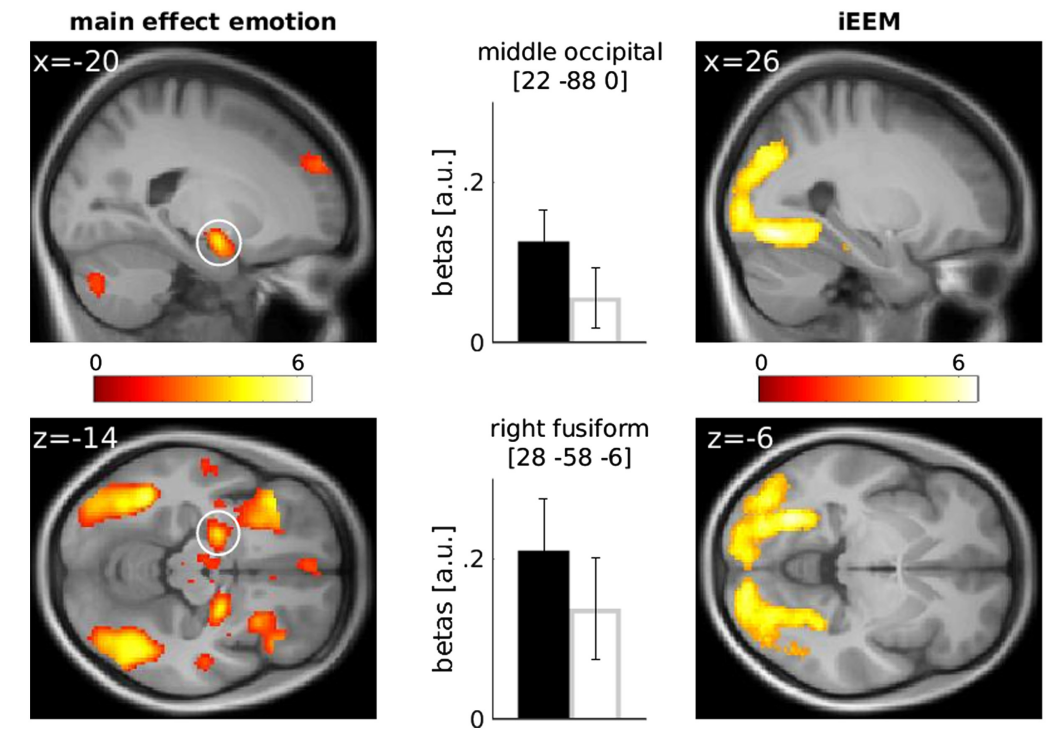
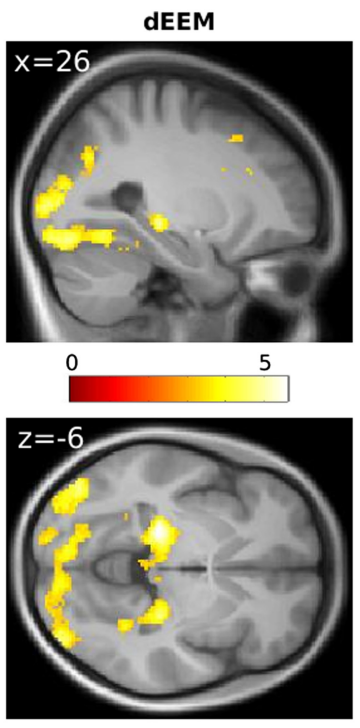

right hippocampus

[28 -34 -2]

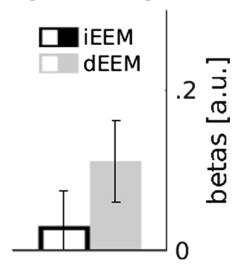

left hippocampus $[-20-28-8]$

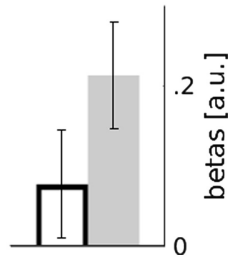

Figure 4. Amygdala coupling related to iEEM and dEEM. (Left panel) The cluster in the left amygdala identified by the main effect of emotion (white circle) served as seed region for the connectivity analyses. (Middle panel) The amygdala was more strongly coupled with the bilateral fusiform, lingual, and middle occipital gyri during successful encoding related to iEEM. (Right panel) The amygdala was more strongly coupled with the bilateral hippocampi, fusiform, lingual, middle occipital, and inferior frontal during processing of negative than neutral pictures that were successfully consolidated. Importantly, the parameter estimates are shown for the peaks that survived the exclusive masking with the opposing EEM contrast and therefore represent the largest observed difference between activities associated with iEEM and dEEM in the given brain areas. The solid bars represent the effect (iEEM or dEEM) that was significant in the given area, the contour bars the effects used as exclusive mask.

which might again be related to the confound that many pictures involved both affect-biased attention and emotional synaptic tagging. However, exclusive masking identified stronger coupling with fusiform and visual areas for iEEM and only with the hippocampus for dEEM. The stronger coupling with visual areas during successful encoding of negative than neutral pictures is consistent with known affect-biased attention effects on visual processing, in particular, the amygdala's modulation of visual activity (Wendt et al. 2011; Chen et al. 2014). In contrast, dEEM was associated with greater coupling with the hippocampus, consistent with amygdala modulation of emotional synaptic tagging (McIntyre et al. 2012). Animal data also show that an electric stimulation of the amygdala after processing neutral objects results specifically in more effective consolidation via enhanced coupling with the hippocampus (Manns and Bass 2016). Similarly, the strength of connectivity of the amygdala with the parahippocampal gyrus during the processing of emotional pictures was associated with greater persistence of emotional memories over time (Ritchey et al. 2008).

Complementing the proposal that the relative independence of the effects of emotional arousal on iEEM and dEEM is due to differences in amygdala activity and coupling, on the neurobiological level, such independence can also be partly explained by the involvement of different adrenoceptor families or subtypes for each EEM (Hein 2006). The role of the $\alpha_{1}$ - and $\beta$-adrenoceptor families, specifically in the effect of emotional arousal on memory consolidation, has been well established by a large body of pharmacological animal and human studies (McIntyre et al. 2012; Lonergan et al. 2013). However, the $\alpha_{2}$-adrenoceptor family might be predominantly involved groups. in processes underlying affect-biased attention. The role of the $\alpha_{2}$-adrenoceptor family has been mainly studied using the $\alpha_{2 \mathrm{~B}}$-adrenoceptor deletion polymorphism, which is associated with a less functional receptor and is moreover in complete linkage disequilibrium with a polymorphism in the promotor of the same gene that results in less transcription (Crassous et al. 2010; Nguyen et al. 2011). Deletion carriers show in one study greater amygdala activity and in another study greater activity in medial prefrontal as well as visual regions during processing of arousing stimuli (Rasch et al. 2009; Todd et al. 2015), an effect that we also found though not for the amygdala. Importantly, deletion carriers show enhanced affect-biased attention (Todd et al. 2013), greater iEEM in free recall (de Quervain et al. 2007) and enhanced recollection specifically for emotionally arousing items, which is consistent with more selective attention during encoding (Todd et al. 2014). In contrast, several studies reported no effect of $\alpha_{2 \mathrm{~B}}$-adrenoceptor genotype on emotional memory consolidation (Naudts et al. 2012; Todd et al. 2014, 2015).
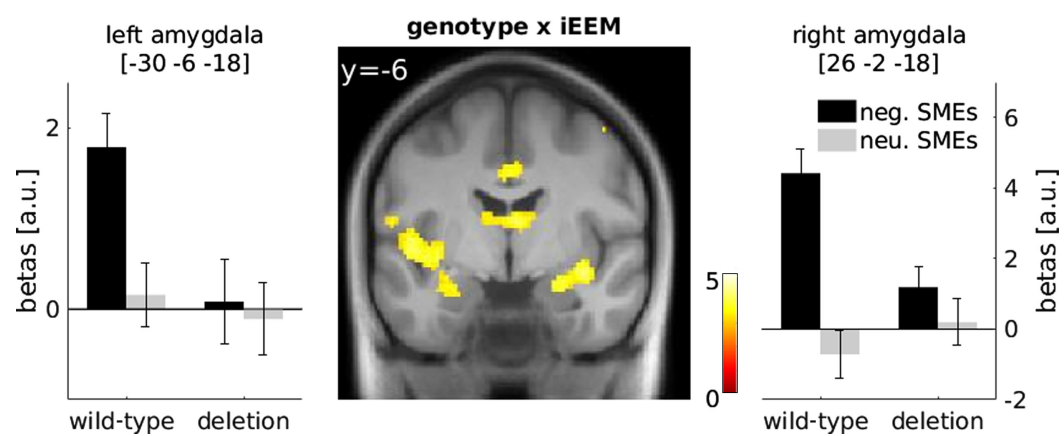

Figure 5. Interaction of $\alpha_{2 \mathrm{~B}}$-adrenocepor genotype and amygdala activity associated with iEEM. Activity in the bilateral amygdala correlated more strongly with $\mathrm{iEEM}$ in wild-type than with $\alpha_{2 \mathrm{~B}}$-adrenocepor deletion carriers. Negative and neutral SMEs for iEEM are shown for both genotype 
In the current and preceding large-scale behavioral study, we failed to replicate the larger iEEM in deletion carriers (as discussed above), but deletion carriers showed a smaller iEEM-related neural activity in the bilateral amygdala and insula. This neural activity difference for iEEM, in particular in the bilateral amygdala, supports the hypothesis that the $\alpha_{2 \mathrm{~B}}$-adrenoceptor is involved in affectbiased attention but not emotional synaptic tagging. Moreover, it is consistent with our proposal based on the results of the whole sample that iEEM involves bilateral amygdala activity (Mackiewicz et al. 2006). However, at first sight, the direction of the difference in neural activity seems surprising because usually a behavioral effect such as larger free recall iEEM in deletion carriers is associated with greater neural activity. Several interpretations of this robust genotype effect on bilateral amygdala, insula, and $\mathrm{mPFC}$ /anterior cingulate activity are possible. One interpretation is that iEEM is the interaction of emotional and neutral SMEs which themselves reflect the increase in activity during encoding that is necessary for successful memory formation. The deletion polymorphism results in fewer and less functional $\alpha_{2 \mathrm{~B}}$-adrenoceptors, which might be expressed also as presynaptic autoreceptors. Therefore, the deletion variant results in an increased noradrenaline release, which explains the greater neural activity with emotional processing we and others have observed. According to the Local Hot Spot model, increase in noradrenaline release results in an enhanced signal-to-noise ratio only at active synapses, which could in turn increase encoding efficiency which might result in a smaller activity increase is necessary for successful encoding (Mather et al. 2015). Alternatively, the $\alpha_{2 \mathrm{~B}}$-adrenoceptor could be expressed predominantly postsynaptically in the amygdala which would result in less activity in response to arousal in the deletion carriers. However, this interpretation does not seem to be supported by the data because the main effect of emotion does not reveal greater activity in wild-type participants in any brain region. Finally, the deletion carriers showed greater emotional processing related activity in the mPFC/anterior cingulate. The MPFC is known to downregulate amygdala activity during emotional processing which could explain the seemingly counterintuitive smaller iEEM in deletion carriers (Kim et al. 2011).

Our replication of an effect of the $\alpha_{2 \mathrm{~B}}$-adrenoceptor polymorphism on activity in the amygdala and other brain regions is also of a more general interest for a characterization of the role of this receptor subtype which was difficult because a selective antagonist was missing. Expression of this subtype was reported so far mostly in the periphery (e.g., vascular tissues) and centrally only in the spinal cord and thalamus. Consistent with this expression pattern, the phenotypes of $\alpha_{2 \mathrm{~B}}$-adrenoceptor knockout mouse were described with respect to physiological parameters such as blood pressure (Bhalla et al. 2013). Only recently, it was shown that the $\alpha_{2 \mathrm{~B}}$-adrenoceptor is more widely expressed in the brain and a role in gating and filtering of incoming sensory information has been suggested based on the behavioral phenotype of the knockout mice (Luhrs et al. 2016). The association of the $\alpha_{2 \mathrm{~B}}$-adrenoceptor polymorphism with effect-biased attention related neural activity is somewhat supportive for this proposed function.

In conclusion, we replicated our previous behavioral finding that iEEM and dEEM where nearly uncorrelated across participants, suggesting these EEMs have distinct neural substrates. Although mean EEMs of the whole sample were of the same magnitude as $3.5 \mathrm{yr}$ ago, only dEEM was weakly correlated across time points, suggesting EEMs are not reliable or stable, at least when assessed by recognition tests. On the neural level, we observed a lateralized amygdala involvement. Specifically, whereas iEEM was associated with bilateral amygdala activity, dEEM was only associated with left and partly more posterior amygdala activity. This is consistent with previous reports of sex- and/or functiondependent amygdala lateralization. During successful immediate emotional memory formation, the amygdala was functionally more coupled with visual areas, consistent with affect-biased attentional modulation of visual processing. During successful delayed memory formation, the amygdala was more functionally coupled with the hippocampus, consistent with emotional synaptic tagging. The relatively lower iEEM-related activity in the bilateral amygdala in deletion carriers suggests that this receptor family is involved in affect-biased attention but not emotional synaptic tagging and is consistent with the proposal that bilateral amygdala is involved in such attention. Taken together, the current data suggest that the processes and underlying neural substrates of iEEM and dEEM differ which would explain their relative independence on the behavioral level. Moreover, the differential activity in the amygdala and hippocampus but also their coupling with other cortical areas might be explained by different adrenoceptor families involved in the different EEMs.

\section{Materials and Methods}

\section{Participants}

Seventy female participants (mean age 28 yr, range 22-38 yr) who had participated in the large behavioral study 3 yr 5 mo earlier on average (range 1 yr 4 mo-5 yr $10 \mathrm{mo}$ ) were recruited. Data acquisition failed for one participant due to equipment malfunction, leaving a sample of 69 . The study was approved by the local ethics committee of the Hamburg Board of Physicians in Germany. All participants signed informed consent and were paid $€ 10$ per hour for participating.

\section{Emotional memory paradigm}

Participants studied 80 neutral and 80 negatively arousing scenes in the MRI scanner. Scenes were drawn from a set of 160 neutral and 160 negative scenes. Only negative, that is no positive, pictures were contrasted with neutral ones to increase statistical power, as negative and positive EEMs partially rely on distinct mechanisms and neural substrates (Talmi et al. 2007; Mickley Steinmetz and Kensinger 2009; Mickley Steinmetz et al. 2010). Each trial consisted of picture presentation $(2 \mathrm{sec})$, followed by rating of the suitability of the picture for a magazine like National Geographic ( $2 \mathrm{sec}$ ), an active baseline task (pointing arrows, 4 $\mathrm{sec})$, and a jittered fixation period $(0.95$ to $3.05 \mathrm{sec})$. This resulted in trial lengths varying from about 9 to $12 \mathrm{sec}$. The trial structure, including the active baseline task, was very similar to what was used in our previous large-scale behavioral study. The presentation rate of the pictures was slow enough to allow PPI analyses (Friston et al. 1997; Gitelman et al. 2003). At the end of this encoding period in the MRI scanner, participants completed $3 \mathrm{~min}$ of a mental rotation task outside of the scanner to clear working memory. They then completed a recognition test with a six-point confidence scale (six high confidence new, one high confidence old) for half of the pictures, randomly intermixed with the same number of unseen pictures as lures. A second recognition test for the remaining pictures followed about $24 \mathrm{~h}$ later. At the conclusion of the study, participants rated the subjective valence and arousal of the pictures on the nine-step Self-Assessment Manikin (Bradley and Lang 1994), which confirmed that the negative pictures were experienced as more negative and more arousing than the neutral ones, with mean valence for negative pictures 2.9 (SD 0.7), neutral 6.6 (SD 1.1), $t_{(68)}=-24,43, P<0.0001$; mean arousal for negative pictures 6.3 (SD 1.2), neutral 2.6 (SD 1.1), $t_{(68)}=23,00, P<0.0001$.

Memory consolidation occurs mostly during slow wave sleep at night and its efficiency is related to sleep spindle number and duration (Dudai et al. 2015). In our previous large-scale behavioral study, sleep duration and quality was assessed using Actiwatches, as well as subjective reports on the German version of the sleep quality scale (SFA). Sleep duration and quality did not correlate with dEEM (duration $r=0.06, P=0.14$; quality $r=0.04, P=0.37$; questionnaire $r=0.02, P=0.69$; unpublished observations). We concluded that these rather superficial measures might not be 
related to the critical variables sleep spindle number and duration. We therefore did not assess sleep duration and quality in the current fMRI study.

\section{Neuroimaging}

Event-related functional whole brain MRI was performed on a 3 Tesla system (Siemens Trio) with a T2*-weighted echo planar imaging sequence in 38 contiguous axial slices (3-mm thickness with 1-mm gap; TR $2.21 \mathrm{sec}$; TE $30 \mathrm{msec}$; flip angle $80^{\circ}$; FOV $216 \times$ 216; matrix $72 \times 72$ ). In order to minimize movement, we acquired four rather short runs of 205 volumes. For spatial normalization, a high-resolution T1-weighted structural MR image was acquired with a 3D MPRAGE sequence (TR $2300 \mathrm{msec}$, TE $2.89 \mathrm{msec}$, flip angle $9^{\circ}, 2401$-mm slices, FOV $256 \times 192$ ).

Statistical parametric mapping (SPM12; Wellcome Department of Imaging Neuroscience) was used to preprocess and analyze the fMRI data. The first five volumes of each run were discarded to account for spin saturation effects. The fMRI data were slice-time corrected, realigned and corrected for susceptibility-by-movement artifacts using the Realign and Unwarp function as implemented in SPM12. T1 images were then coregistered to the individual mean functional image, segmented and normalized using the DARTEL toolbox to create individual flow fields. Flow fields were used to normalize the functional images to MNI space. Finally, images were smoothed with a full-width half-maximum Gaussian kernel of $8 \mathrm{~mm}$.

The four runs of each participant were concatenated for the first-level models. Each participant's fMRI data was modeled using a general linear model (GLM) with the following regressors: two emotional arousal (neutral versus negative) $\times 2$ subsequent memory (subsequent hits versus misses) $\times 2$ delay period (immediate versus delayed). Regressors were created by convolving the respective onset vectors (i.e., in an event-related manner) with the canonical hemodynamic response function. Run-specific constants were included in the model. To remove movement-related artifacts, nuisance regressors were created for images with movements $>0.2$ $\mathrm{mm} / \mathrm{TR}$. No participant had to be excluded due to excessive movement. The time series were corrected for baseline drifts by applying a high-pass filter (128 sec) and for serial dependency using an AR(1) autocorrelation model. Both corrections were done within runs. The $\beta$ images corresponding to the regressors of interest for all participants were then included in a group-level GLM. Some participants had none or only few trials in one of the immediate miss conditions. We did not exclude these participants from the second-level analysis because this would have biased the sample toward participants with worse memory performance. However, the variance of the parameter estimates of these conditions was expected to be higher, thus violating the i.i.d.-assumption. Unequal variances were dealt with by using the nonsphericity correction, as implemented in the flexible factorial second level model of SPM.

We adopted both an ROI- as well as a peak-based approach to characterize brain activity associated with iEEM and dEEM. In the ROI-based approach, mean parameter estimates were calculated from all voxels in anatomical masks of the left and right amygdala and the left and right hippocampus for all conditions. The iEEMs (interaction of arousal and subsequent memory success for immediately tested pictures) and dEEMs (the same interaction for the delayed tested pictures) were then contrasted outside of SPM in a GLM with the within-subjects factors of brain structure (amygdala versus hippocampus), hemisphere, and delay.

The peak voxel-based approach was conducted to gain spatial specificity within the ROIs, e.g., to potentially differentiate different response profiles with the amygdala, and to facilitate whole brain analyses. High spatial specificity could be critical in differentiating the neural correlates of iEEM and dEEM because of the previously mentioned design-inherent challenge that many of the immediately tested items would have been remembered also on the next day and vice versa. Areas where activity correlated with emotion irrespective of encoding success were identified by the main effect of emotion, which reflected processing of negative and neutral pictures. To identify areas where activity correlated with iEEM, the interaction of encoding success and emotional arousal was computed for the immediately tested pictures, as was done for the ROI analyses. The same interaction was similarly computed for dEEM by taking pictures tested after $24 \mathrm{~h}$. In order to identify areas predominantly involved in the immediate versus delayed EEM, we computed the interactions between IEEM and dEEM. However, to deal with the complication that both processes are innate to the affective processing of many pictures, making their separation difficult even with large samples, we also used a masking approach where we exclusively masked each contrast with the other (using a liberal threshold of $P<0.05$ uncorrected). To locate areas where activity is associated with both iEEM and dEEM, we performed a conjunction analysis.

PPI analyses were conducted, as implemented in SPM12 (Friston et al. 1997), to assess differences in the functional coupling of the amygdala between successful memory formation of negative and neutral pictures. We specifically tested with which brain areas was the part of the amygdala identified in emotional processing (seed region: main effect of emotion threshold at $P<0.001$, peak $\mathrm{xyz}=\left[\begin{array}{l}-20-6-14 \\ \mathrm{w}\end{array}, \mathrm{Z}=\mathrm{inf}, 243\right.$ voxels) more strongly coupled when processing negative rather than neutral pictures during successful immediate and delayed memory formation. The first eigenvariate of the time series was extracted from the amygdala seed region for the immediate and delayed test and as well as for the interaction of successful encoding of negative versus neutral pictures, after adjusting for effects of no interest (including session constants and high-pass filter). These two time series were fed into new first-level models similar to the above described ones as additional regressors, and the parameter estimates of the interaction regressors were used in a second-level analysis. Again, an exclusive masking procedure $(P<0.05$, uncorrected) was adopted in order to identify areas more strongly coupled for immediate rather than delayed EEM and vice versa.

To assess genotype-dependent differences in brain activity, we contrasted deletion carriers (homo- and heterozygote) versus homozygote wild-type for the $\alpha_{2 \mathrm{~B}}$-noradrenergic receptor polymorphism by adding genotype group as a factor to the second-level analysis (Rasch et al. 2009; Urner et al. 2011; Todd et al. 2015). Interactions between genotype and the main effect of emotion, as well as with the interaction contrasts representing iEEM and dEEM, were computed.

All voxel coordinates are given in MNI space. Results of all analyses were considered significant at $P<0.05$, peak voxel familywise error corrected for multiple comparisons within previously reported regions involved in EEM. We hypothesized that the primary brain regions associated with the EEMs would be the amygdala, and hippocampus as well as for the PPI analyses the fusiform, lingual, and middle occipital gyrus (Kensinger and Schacter 2007; Ritchey et al. 2011). Bilateral anatomical masks were created from the Automatic Anatomic Labeling toolbox for small volume correction (Tzourio-Mazoyer et al. 2002). Peaks of activity that survive family-wise error correction for the entire scan volume are reported. Finally, for exploratory reasons and to allow for subsequent meta analyses (Hayasaka and Nichols 2004), we also report peaks at a more lenient voxelwise statistical threshold of $P<0.001$, uncorrected for multiple comparisons, and in a cluster with minimum 10 voxels are also listed (and clearly marked) in the tables.

\section{Acknowledgments}

We would like to thank Gina Joue for her constructive comments that helped to improve the manuscript.

\section{References}

Barnacle GE, Montaldi D, Talmi D, Sommer T. 2016. The list-composition effect in memory for emotional and neutral pictures: differential contribution of ventral and dorsal attention networks to successful encoding. Neuropsychologia 90: 125-135.

Bayer J, Schultz H, Gamer M, Sommer T. 2014. Menstrual-cycle dependent fluctuations in ovarian hormones affect emotional memory. Neurobiol Learn Mem 110: 55-63. 
Bayer J, Gläscher J, Finsterbusch J, Schulte LH, Sommer T. 2018. Linear and inverted U-shaped dose-response functions describe estrogen effects on hippocampal activity in young women. Nat Commun 9: 1220 .

Bergado JA, Lucas M, Richter-Levin G. 2011. Emotional tagging-A simple hypothesis in a complex reality. Prog Neurobiol 94: 64-76.

Bhalla S, Ali I, Andurkar SV, Gulati A. 2013. Centhaquin antinociception in mice is mediated by $\alpha 2 \mathrm{~A}$ - and $\alpha 2 \mathrm{~B}$ - but not $\alpha 2 \mathrm{C}$-adrenoceptors. Eur J Pharmacol 715: 328-336.

Bradley MM, Lang PJ. 1994. Measuring emotion: the self-assessment manikin and the semantic differential. J Behav Ther Exp Psychiatry 25: 49-59.

Cahill L, Haier RJ, White NS, Fallon J, Kilpatrick L, Lawrence C, Potkin SG, Alkire MT. 2001. Sex-related difference in amygdala activity during emotionally influenced memory storage. Neurobiol Learn Mem 75: 1-9.

Cahill L, Uncapher M, Kilpatrick L, Alkire MT, Turner J. 2004. Sex-related hemispheric lateralization of amygdala function in emotionally influenced memory: an FMRI investigation. Learn Mem 11: 261-266.

Canli T, Desmond JE, Zhao Z, Gabrieli JD. 2002. Sex differences in the neural basis of emotional memories. Proc Natl Acad Sci 99: 10789-10794.

Chen Y, Li H, Jin Z, Shou T, Yu H. 2014. Feedback of the amygdala globally modulates visual response of primary visual cortex in the cat. Neuroimage 84: $775-785$.

Crassous P-A, Blaise R, Marquette A, Snapir A, Scheinin M, Paris H, Schaak S. 2010. Identification of a novel 12-nucleotide insertion polymorphism in the promoter region of ADRA2B: full linkage with the 9-nucleotide deletion in the coding region and influence on transcriptional activity. Biochem Pharmacol 79: 407-412.

de Quervain DJ-F, Kolassa I-T, Ertl V, Onyut PL, Neuner F, Elbert T, Papassotiropoulos A. 2007. A deletion variant of the $\alpha 2 \mathrm{~b}$-adrenoceptor is related to emotional memory in Europeans and Africans. Nat Neurosci 10: $1137-1139$.

Dolcos F, Denkova E, Dolcos S. 2012. Neural correlates of emotional memories: a review of evidence from brain imaging studies. Psychologia 55: $80-110$.

Dougal S, Rotello CM. 2007. "Remembering" emotional words is based on response bias, not recollection. Psychon Bull Rev 14: 423-429.

Dudai Y, Karni A, Born J. 2015. The consolidation and transformation of memory. Neuron 88: 20-32.

Friston KJ, Buechel C, Fink GR, Morris J, Rolls E, Dolan RJ. 1997. Psychophysiological and modulatory interactions in neuroimaging. Neuroimage 6: 218-229.

Gitelman DR, Penny WD, Ashburner J, Friston KJ. 2003. Modeling regional and psychophysiologic interactions in fMRI: the importance of hemodynamic deconvolution. Neuroimage 19: 200-207.

Hagena H, Hansen N, Manahan-Vaughan D. 2016. $\beta$-adrenergic control of hippocampal function: subserving the choreography of synaptic information storage and memory. Cereb Cortex 26: 1349-1364.

Hamann S. 2001. Cognitive and neural mechanisms of emotional memory. Trends Cogn Sci 5: 394-400.

Hayasaka S, Nichols TE. 2004. Combining voxel intensity and cluster extent with permutation test framework. Neuroimage 23: 54-63.

Hein L. 2006. Adrenoceptors and signal transduction in neurons. Cell Tissue Res 326: 541-551.

Inman CS, Manns JR, Bijanki KR, Bass DI, Hamann S, Drane DL, Fasano RE, Kovach CK, Gross RE, Willie JT. 2017. Direct electrical stimulation of the amygdala enhances declarative memory in humans. Proc Natl Acad Sci 115: $98-103$

Johnson RT, Breedlove SM, Jordan CL. 2008. Sex differences and laterality in astrocyte number and complexity in the adult rat medial amygdala. J Comp Neurol 511: 599-609.

Johnson RT, Schneider A, DonCarlos LL, Breedlove SM, Jordan CL. 2012. Astrocytes in the rat medial amygdala are responsive to adult androgens. J Comp Neurol 520: 2531-2544.

Kang C, Wang Z, Surina A, Lü W. 2014. Immediate emotion-enhanced memory dependent on arousal and valence: the role of automatic and controlled processing. Acta Psychol (Amst) 150: 153-160.

Kensinger EA, Corkin S. 2004. Two routes to emotional memory: distinct neural processes for valence and arousal. Proc Natl Acad Sci 101: 3310-3315.

Kensinger EA, Schacter DL. 2007. Remembering the specific visual details of presented objects: neuroimaging evidence for effects of emotion. Neuropsychologia 45: 2951-2962.

Kilpatrick L, Cahill L. 2003. Amygdala modulation of parahippocampal and frontal regions during emotionally influenced memory storage. Neuroimage 20: 2091-2099.

Kim MJ, Loucks RA, Palmer AL, Brown AC, Solomon KM, Marchante AN, Whalen PJ. 2011. The structural and functional connectivity of the amygdala: from normal emotion to pathological anxiety. Behav Brain Res 223: 403-410.

Koen JD, Barrett FS, Harlow IM, Yonelinas AP. 2016. The ROC Toolbox: a toolbox for analyzing receiver-operating characteristics derived from confidence ratings. Behav Res Methods 49: 1399-1406.
Kohno S, Noriuchi M, Iguchi Y, Kikuchi Y, Hoshi Y. 2015. Emotional discrimination during viewing unpleasant pictures: timing in human anterior ventrolateral prefrontal cortex and amygdala. Front Hum Neurosci 9: 51.

Lonergan M, Olivera-Figueroa L, Pitman R, Brunet A. 2013. Propranolol's effects on the consolidation and reconsolidation of long-term emotional memory in healthy participants: a meta-analysis. J Psychiatry Neurosci 38: 222-231.

Luhrs L, Manlapaz C, Kedzie K, Rao S, Cabrera-Ghayouri S, Donello J, Gil D. 2016. Function of brain $\alpha 2 \mathrm{~B}$-adrenergic receptor characterized with subtype-selective $\alpha 2 \mathrm{~B}$ antagonist and $\mathrm{KO}$ mice. Neuroscience 339: 608-621.

Mackiewicz KL, Sarinopoulos I, Cleven KL, Nitschke JB. 2006. The effect of anticipation and the specificity of sex differences for amygdala and hippocampus function in emotional memory. Proc Natl Acad Sci 103: 14200-14205.

Manns JR, Bass DI. 2016. The amygdala and prioritization of declarative memories. Curr Dir Psychol Sci 25: 261-265.

Markovic J, Anderson AK, Todd RM. 2014. Tuning to the significant: neural and genetic processes underlying affective enhancement of visual perception and memory. Behav Brain Res 259: 229-241.

Mather M, Clewett D, Sakaki M, Harley CW. 2015. Norepinephrine ignites local hot spots of neuronal excitation: How arousal amplifies selectivity in perception and memory. Behav Brain Sci 39: e200.

McClearn GE, Johansson B, Berg S, Pedersen NL, Ahern F, Petrill SA, Plomin R. 1997. Substantial genetic influence on cognitive abilities in twins 80 or more years old. Science 276: $1560-1563$.

McIntyre CK, McGaugh JL, Williams CL. 2012. Interacting brain systems modulate memory consolidation. Neurosci Biobehav Rev 36: 1750-1762.

Mickley Steinmetz KR, Kensinger EA. 2009. The effects of valence and arousal on the neural activity leading to subsequent memory. Psychophysiology 46: 1190-1199.

Mickley Steinmetz KR, Addis DR, Kensinger EA. 2010. The effect of arousal on the emotional memory network depends on valence. Neuroimage 53: 318-324.

Mickley Steinmetz KR, Schmidt K, Zucker HR, Kensinger EA. 2012. The effect of emotional arousal and retention delay on subsequent-memory effects. Cogn Neurosci 3: 150-159.

Naudts K, Azevedo R, David A, van Heeringen C, Gibbs A. 2012. Influence of COMT val158met and ADRA2B deletion polymorphisms on recollection and familiarity components of human emotional memory. J Psychopharmacol (Oxf) 26: 819-829.

Nguyen K, Kassimatis T, Lymperopoulos A. 2011. Impaired desensitization of a human polymorphic $\alpha 2 \mathrm{~B}$-adrenergic receptor variant enhances its sympatho-inhibitory activity in chromaffin cells. Cell Commun Signal 9: 5 .

Panizzon MS, Lyons MJ, Jacobson KC, Franz CE, Grant MD, Eisen SA, Xian H, Kremen WS. 2011. Genetic architecture of learning and delayed recall: a twin study of episodic memory. Neuropsychology 25: 488-498.

Payne JD, Stickgold R, Swanberg K, Kensinger EA. 2008. Sleep preferentially enhances memory for emotional components of scenes. Psychol Sci 19: 781-788.

Pfau DR, Hobbs NJ, Breedlove SM, Jordan CL. 2016. Sex and laterality differences in medial amygdala neurons and astrocytes of adult mice: sex and laterality differences in MePD. J Comp Neurol 524: 2492-2502.

Phillips ML, Medford N, Young AW, Williams L, Williams SC, Bullmore ET, Gray JA, Brammer MJ. 2001. Time courses of left and right amygdalar responses to fearful facial expressions. Hum Brain Mapp 12: 193-202.

Rasch B, Spalek K, Buholzer S, Luechinger R, Boesiger P, Papassotiropoulos A, de Quervain DJ-F. 2009. A genetic variation of the noradrenergic system is related to differential amygdala activation during encoding of emotional memories. Proc Natl Acad Sci 106: 19191-19196.

Ritchey M, Dolcos F, Cabeza R. 2008. Role of amygdala connectivity in the persistence of emotional memories over time: an event-related FMRI investigation. Cereb Cortex 18: 2494-2504.

Ritchey M, LaBar KS, Cabeza R. 2011. Level of processing modulates the neural correlates of emotional memory formation. J Cogn Neurosci 23: 757-771.

Schümann D, Bayer J, Talmi D, Sommer T. 2017. Dissociation of immediate and delayed effects of emotional arousal on episodic memory. Neurobiol Learn Mem 148: 11-19.

Sergerie K, Chochol C, Armony JL. 2008. The role of the amygdala in emotional processing: a quantitative meta-analysis of functional neuroimaging studies. Neurosci Biobehav Rev 32: 811-830.

Sharot T, Yonelinas AP. 2008. Differential time-dependent effects of emotion on recollective experience and memory for contextual information. Cognition 106: 538-547.

Sharot T, Verfaellie M, Yonelinas AP. 2007. How emotion strengthens the recollective experience: a time-dependent hippocampal process. PLoS One 2: e1068. 
Sommer T, Glascher J, Moritz S, Buchel C. 2008. Emotional enhancement effect of memory: removing the influence of cognitive factors. Learn Mem 15: 569-573.

Talmi D, Schimmack U, Paterson T, Moscovitch M. 2007. The role of attention and relatedness in emotionally enhanced memory. Emotion 7: 89-102.

Talmi D, Lohnas L, Daw N. 2017. A retrieved context model of the emotional modulation of memory. bioRxiv doi: 10.1101/175653.

Todd RM, Palombo DJ, Levine B, Anderson AK. 2011. Genetic differences in emotionally enhanced memory. Neuropsychologia 49: 734-744.

Todd RM, Muller DJ, Lee DH, Robertson A, Eaton T, Freeman N, Palombo DJ, Levine B, Anderson AK. 2013. Genes for emotion-enhanced remembering are linked to enhanced perceiving. Psychol Sci 24: 2244-2253.

Todd RM, Müller DJ, Palombo DJ, Robertson A, Eaton T, Freeman N, Levine B, Anderson AK. 2014. Deletion variant in the ADRA2B gene increases coupling between emotional responses at encoding and later retrieval of emotional memories. Neurobiol Learn Mem 112: 222-229.

Todd RM, Ehlers MR, Muller DJ, Robertson A, Palombo DJ, Freeman N, Levine B, Anderson AK. 2015. Neurogenetic variations in norepinephrine availability enhance perceptual vividness. J Neurosci 35: 6506-6516.

Tzourio-Mazoyer N, Landeau B, Papathanassiou D, Crivello F, Etard O, Delcroix N, Mazoyer B, Joliot M. 2002. Automated anatomical labeling of activations in SPM using a macroscopic anatomical parcellation of the MNI MRI single-subject brain. Neuroimage 15: 273-289.

Urner M, van Wingen G, Franke B, Rijpkema M, Fernández G, Tendolkar I. 2011. Genetic variation of the $\alpha 2 b$-adrenoceptor affects neural correlates of successful emotional memory formation. Hum Brain Mapp 32: 2096-2103.

Wendt J, Weike AI, Lotze M, Hamm AO. 2011. The functional connectivity between amygdala and extrastriate visual cortex activity during emotional picture processing depends on stimulus novelty. Biol Psychol 86: 203-209. Wright CI, Fischer H, Whalen PJ, McInerney SC, Shin LM, Rauch SL. 2001. Differential prefrontal cortex and amygdala habituation to repeatedly presented emotional stimuli. Neuroreport 12: 379-383.

Yonelinas AP. 2002. The nature of recollection and familiarity: a review of 30 years of research. J Mem Lang 46: 441-517.

Received December 22, 2017; accepted in revised form April 4, 2018. 


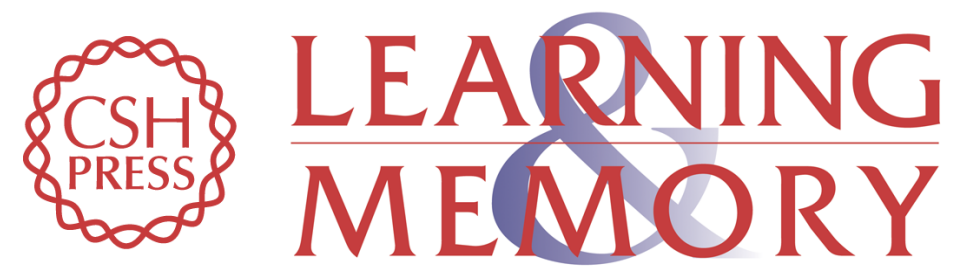

\section{Dissociable contributions of the amygdala to the immediate and delayed effects of emotional arousal on memory}

Dirk Schümann and Tobias Sommer

Learn. Mem. 2018, 25:

Access the most recent version at doi:10.1101/Im.047282.117

\begin{aligned} & \hline References $\begin{array}{l}\text { This article cites } 67 \text { articles, } 10 \text { of which can be accessed free at: } \\ \text { http://learnmem.cshlp.org/content/25/6/283.full.html\#ref-list-1 }\end{array} \\ & \begin{array}{r}\text { Creative } \\ \text { Commons } \\ \text { License }\end{array} \begin{array}{l}\text { This article is distributed exclusively by Cold Spring Harbor Laboratory Press for the } \\ \text { first } 12 \text { months after the full-issue publication date (see } \\ \text { http://learnmem.cshlp.org/site/misc/terms.xhtml). After } 12 \text { months, it is available under } \\ \text { a Creative Commons License (Attribution-NonCommercial } 4.0 \text { International), as } \\ \text { described at http://creativecommons.org/licenses/by-nc/4.0/. }\end{array} \\ & \begin{array}{c}\text { Receive free email alerts when new articles cite this article - sign up in the box at the } \\ \text { top right corner of the article or click here. }\end{array} \\ & \begin{array}{c}\text { Service } \\ \text { Serting }\end{array}\end{aligned}$ 